\title{
Evaluation of the Antioxidants as Adjunct Therapy in Cattle Naturally Infected with Bovine Tropical Theileriosis
}

\author{
Neelam $^{1 *}$, Naresh Kumar Rakha ${ }^{1}$, Ricky Jhambh ${ }^{1}$, Meenakshi Virmani', \\ Parveen Goel ${ }^{1}$ and Ramkaran ${ }^{2}$ \\ ${ }^{1}$ Department of Veterinary Medicine, LLR University of Veterinary and Animal Sciences, \\ Hisar - 125004, Haryana, India \\ ${ }^{2}$ Department of Veterinary Physiology and Biochemistry, LLR University of Veterinary and \\ Animal Sciences, Hisar - 125004, Haryana, India \\ *Corresponding author
}

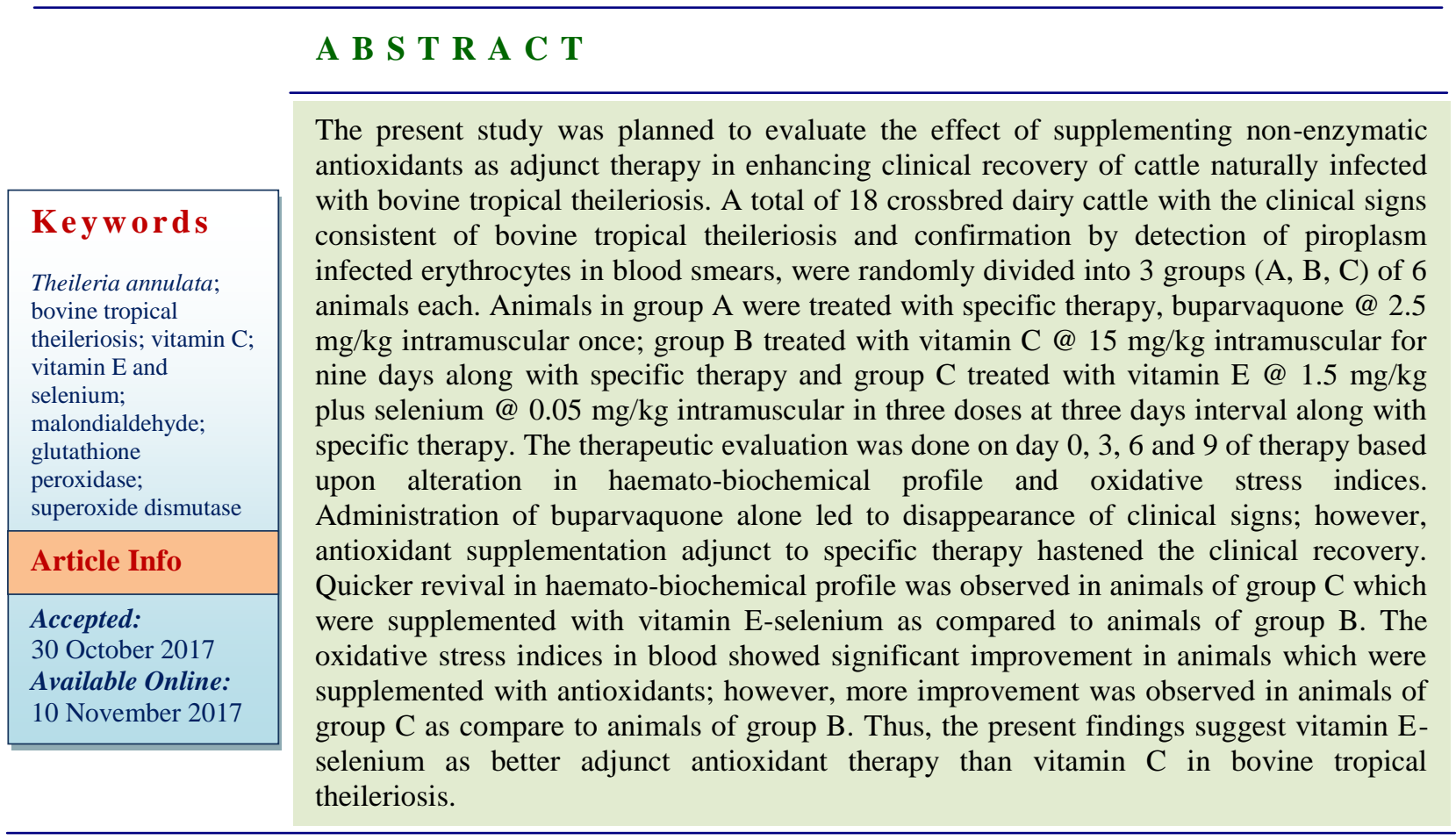

\section{Introduction}

Bovine tropical theileriosis, a disease of global economic importance, caused by heamoprotozoan parasite Theileria annulata and transmitted by ticks of genus Hyalomma (Preston, 2001) is characterized by lymphadenopathy, spleenomegaly, fever, anaemia, weakness and loss of body weight
(Omer et al., 2002; El-Deeb and Younis, 2009). A significant feature of the disease is anaemia due to overproduction of cytokines and reactive oxygen species (Nazifi et al., 2009; Saleh et al., 2011), haematopoietic precursor cell destruction (Mbassa et al., 1994), activated complement products (Omer 
et al., 2002), binding of autoantibody ( $\operatorname{IgG}$ ) to red blood cells (RBC) and removal of infected and non-infected erythrocytes by phagocytosis (Shiono et al., 2004). Hepatic tissue damage in this disease includes coagulative necrosis, destruction of hepatic cords and heavy infiltration of lymphocytes in peri-portal area, which indicates severe damage to hepatobiliary system due to hypoxia resulting from anaemia and jaundice (Stockham et al., 2000).

Oxidative stress due to increase in reactive oxygen species in cells of hosts infected with parasite $T$. annulata is well established (Grewal et al., 2005; Nazifi et al., 2008; ElDeeb and Younis, 2009). Oxidative stress evident by the reduction in activity of antioxidant enzymes and decreased level of antioxidant vitamins in blood of parasitized animals has been reported in theileria infected cattle (Issi and Gul, 2001; Shiono et al., 2001; Nazifi et al., 2008). Reactive oxygen species can be scavenged by antioxidant system which includes antioxidant enzymes glutathione peroxidase and superoxide dismutase and non-enzymatic components involving vitamin $\mathrm{E}$, vitamin $\mathrm{C}$, selenium and glutathione. $\alpha$-tocopherol (vitamin E) and ascorbic acid (vitamin C) acts as cellular antioxidant vitamins which are present in the cell membrane and plasma lipoproteins (Bast et al., 1991).

The antioxidant mechanisms of ascorbic acid are based on the donation of hydrogen atom to lipid radicals, quenching of singlet oxygen, and removal of molecular oxygen (Rumsey et al., 1999). Vitamin E effectively minimizes oxidative stress, lipid peroxidation and toxic effects of reactive oxygen species in biological systems (Ogutcu et al., 2006). Selenium (Se) is component of some proteins and enzymes present in blood and tissues and acts as a potent antioxidant as well as potent immunomodulator. These protective effects of
Se (as co-antioxidant) seem to be primarily associated with its presence in the selenoenzymes, which are known to protect DNA and other cellular components from oxidative damage (Valko et al., 2006).

Buparvaquone is the most effective and safest drug for treatment of Theileria in cattle, and this drug has been thoroughly investigated both in vitro and in vivo (Dhar et al., 1988; McHardy, 1990; Keles et al., 2001). Kumar et al., (2016) measured the oxidative stress in Theileria infected cattle and reported that there were significantly altered levels of enzymes indicating a high degree of oxidative stress in theileria infected animals.

Further, the administration of buparvaquone (drug of choice) alone led to further increase in levels of oxidative stress. Study for the role of antioxidant therapy along with anti theilerial drug for three days in bovine tropical theileriosis revealed that there was significant reduction in oxidative stress levels and there was faster clinical recovery in infected-animals. However, when the vitamin $\mathrm{C}$ administration was stopped after 3 days, oxidative stress levels again rose beyond normal values.

So keeping in view these observations, the present study was planned to evaluate the effect of supplementing non enzymatic antioxidants and that too given for a longer duration to further enhance clinical recovery of theileriosis in bovines.

\section{Materials and Methods}

The study was conducted in 18 crossbred (9 adults and 9 young) dairy cattle naturally infected with $T$. annulata. Animals found infected with theileriosis in laboratory diagnosis, not given any prior treatment and haemoglobin level $>6 \mathrm{~g} / \mathrm{dl}$ were selected for therapeutic evaluation. 


\section{Therapeutics regimen}

T. annulata infected animals were randomly divided into three groups. Group A $(n=6)$ animals treated with buparvaquone only @ $2.5 \mathrm{mg} / \mathrm{kg}$ body weight intramuscular once, group $\mathrm{B}(\mathrm{n}=6)$ animals treated with buparvaquone @2.5 mg/kg body weight intramuscular once and ascorbic acid @ $15 \mathrm{mg} / \mathrm{kg}$ body weight intramuscular daily for 9 days and group $C(n=6)$ animals treated with buparvaquone @ $2.5 \mathrm{mg} / \mathrm{kg}$ body weight intramuscular once with vitamin E @ $1.5 \mathrm{mg} / \mathrm{kg}$ body weight and selenium @ $0.05 \mathrm{mg} / \mathrm{kg}$ body weight intramuscular on day 0,3 and 6.

The samples were collected on day $0,3,6$ and 9 to monitor - clinical recovery, presence/absence of a clinical sign and effect of supplementing antioxidant therapy on recovery process.

\section{Clinical observations}

Clinical vital parameters rectal temperature $\left({ }^{\circ} \mathrm{F}\right)$, pulse rate (per minute) and respiration rate (per minute) were recorded. The superficial lymph node(s) enlargement graded as 1 to 4 .. The lymph node enlargement was graded as 1,2, 3 or 4 corresponding to 'very small', 'small', 'large', or 'very large' lymph nodes respectively (Rakha and Sharma, 2003).

\section{Sampling}

Blood samples were collected in triplet, in ethylenediamine-tetraacetic acid (EDTA) vial for haematological examination, in vial without anticoagulant for harvesting serum and in centrifuge tube containing heparin for separation of plasma and haemolysate. The plasma was separated in aliquots and 10\% stock haemolysate was prepared form blood containing heparin. The plasma, haemolysate and serum were stored at $-20^{\circ} \mathrm{C}$ in aliquots till analysis.

\section{Parasitological observations}

Thin blood smears and lymph node aspirate smears from swollen prescapular lymph nodes were fixed in methanol at the site of sample collection and were stained by Giemsa stain using 1:10 dilution for $30 \mathrm{~min}$. Percent parasitaemia was estimated microscopically by counting the numbers of piroplasm infected erythrocyte in total of about 1,000 erythrocytes.

Presence of schizonts in biopsy smears was semi-quantitatively measured on the scale of 1 to 4 . Wherein ' 1 ' stands for rare; ' 2 ' means sparse; ' 3 ' means high and '4' means very high in numbers in the smear.

\section{Haemato-biochemical examinations}

The blood samples collected in EDTA vials were used for complete haematological examination using fully automated Haematology Cell Counter (MS4s, Melet Schlosing Lab.). The serum samples were analyzed for estimation of biochemical profile using fully automated random access Clinical Chemistry Analyzer (EM Destiny 180, Erba Diagnostics Mannheim GmbH). Blood samples collected in heparin were used for measurement of oxidative stress indices both in plasma and haemolysate. Lipid peroxidation in terms of malondialdehyde (MDA) levels was assessed by method of Ohkawa et al., (1979). Glutathione peroxidase (GPx) activity was measured by method of Hafeman et al., (1974). Superoxide dismutase (SOD) activity was measured by method of Madesh and Balsubramanian (1998). The haemoglobin in the haemolysate was estimated spectrophotometrically by the cyanomethemoglobin method (Vankampen and Ziglstra, 1961). 


\section{Statistical analysis}

The data generated was analyzed statistically by suitable statistical methods using statistical software package (SPSS 16.0). For analysis of various parameters observed for therapeutic efficacy, within and between groups, two-way analysis of variance (ANOVA) was applied. The results are presented as Mean \pm S.E. at the significance level, $\mathrm{p} \leq 0.05$.

\section{Results and Discussion}

The present study was planned and conducted to check the hypothesis that oxidative stress and liver damage caused by $T$. annulata need to be reversed to ensure faster and complete clinical recovery and administration of different antioxidants should result into mitigation of damage caused by free radicals during the oxidative stress.

\section{Clinical profile}

Monitoring of clinical profile is depicted in table 1. All animals which were anorectic on day zero started feeding except one animal in group A which started feeding on day 6 . Diarrhoea was present in three animals in group A, four animals in group B and $\mathrm{C}$ each. In animals of group $\mathrm{B}$ and group $\mathrm{C}$ there was no diarrhoea on day 3 , while one animal in group A was diarrhoeic on day 3 and recovered from diarrhoea on day 6. All animals were dull on day 0 . There was slow improvement and on day 9 three animals in group A, one animal in group B and one animal in group $\mathrm{C}$ were dull. Recovery in terms of anorexia, diarrhoea and dullness was faster in animals of group B and group C as compare to animals of group A. Clinical recovery observed in the present study was similar to that reported by Kumar et al., (2016). There was almost no difference in recovery and clinical profile parameters in animals of group B and group C, but the recovery was faster in these animals as compare to animals of group A.

\section{Clinical Vital parameters}

Clinical vital parameters are depicted in table 2. After 3 days of buparvaquone treatment there was significant $(p \leq 0.05)$ reduction in rectal temperature in all the animals and remained normal up to 9 days. As far as body temperature of infected animals is concerned, administration of antioxidants, vitamin $\mathrm{C}$ and vitamin $\mathrm{E}$ along with selenium did not make any significant impact. In our findings reduction in rectal temperature was due to the specific drug called buparvaquone alone, since no antipyretic/analgesic was administered to infected animals. Kumar et al., (2016) also reported reduction in temperature due to buparvaquone alone in cattle infected with $T$. annulata.

Pulse rate on day 3 increased non significantly $(p \leq 0.05)$ in animals of group A and decreased significantly in animals of group B and C. From day 3 to day 9, there was continuous significant decrease pulse rate of animals in all the three groups. Respiration rate increased significantly in animals of group $\mathrm{A}$ and decreased significantly in animals of group $\mathrm{C}$ and non-significantly in animals of group B. Maximum significant decrease among these three groups was observed in group C, followed by group B and least in group A. It may be due to decrease in harmful pathogenic effect of $T$. annulata and reduction in degree of anaemia.

Gradual reduction in size of lymph node in all the theileria infected animals was observed from day 0 to day 9 , however lymph nodes remained palpable throughout the course of study. It is to be mentioned here that in all the animals which have suffered from bovine tropical theileriosis previously, lymph nodes always remained palpable (Rakha and 
Sharma, 2003; Kumar et al., 2016). As administration of theilericidal drug buparvaquone, kills all Koch's blue bodies and consequently regression of lymph node is faster. Additional supplementation of antioxidant therapy did not make additional measurable impact on size of lymph node(s).

\section{Parasitological observations}

Percent parasitemia in blood smear of 18 diseased animals was $0.42 \pm 0.04$ and schizont density was $0.61 \pm 0.20$. The results were in agreement with observations of AlTemeimy (1982), Al-Robayi (1999) and Stockham et al., (2000). After administration of specific drug buparvaquone, animals of all groups were found to be free from schizonts and piroplasm on day 3 and remained so up to day 9. It revealed that buparvaquone administration killed all schizonts and piroplasm of $T$. annulata. This observation is in agreement with earlier reports of Sharma $e t$ al., (1987) and Singh (1990).

\section{Haematological examinations}

Erythrocytic indices are depicted in table 3. Hematological parameters viz. Hb, TEC, PCV and TLC revealed non-significant $(p \leq 0.05)$ changes after administration of therapy. It might be due to our selection of mild cases of theileriosis in this study. However the significant changes in relative leucocytes count in the group B and group C were found up on administration of antioxidant therapy. Decrease in relative lymphocyte count may be due to removal of infected lymphocytes by phagocytic system. Increase in neutrophil count could be related to the protection of cell membrane and intracellular organelles by the antioxidant effect (Smith et al., 1997).

Table.1 Changes in Clinical profile in cattle infected with $T$. annulata during 9 days of treatment period

\begin{tabular}{|c|c|c|c|c|}
\hline \multirow[t]{2}{*}{ Clinical sign } & \multirow[t]{2}{*}{$\begin{array}{l}\text { Day post } \\
\text { treatment }\end{array}$} & \multicolumn{3}{|c|}{$\begin{array}{l}\text { No. of Diseased animals depicting clinical sign during } 9 \text { days } \\
\text { of study period }\end{array}$} \\
\hline & & Group A (n=6) & Group B $(\mathrm{n}=6)$ & Group C (n=6) \\
\hline \multirow[t]{4}{*}{ Anorexia } & $\mathrm{d} 0$ & $6 / 6$ & $6 / 6$ & $6 / 6$ \\
\hline & d 3 & $1 / 6$ & $0 / 6$ & $0 / 6$ \\
\hline & $\mathrm{d} 6$ & $0 / 6$ & $0 / 6$ & $0 / 6$ \\
\hline & $\mathrm{d} 9$ & $0 / 6$ & $0 / 6$ & $0 / 6$ \\
\hline \multirow[t]{4}{*}{ Diarrhoea } & $\mathrm{d} 0$ & $3 / 6$ & $4 / 6$ & $4 / 6$ \\
\hline & d 3 & $1 / 6$ & $0 / 6$ & $0 / 6$ \\
\hline & $\mathrm{d} 6$ & $0 / 6$ & $0 / 6$ & $0 / 6$ \\
\hline & d 9 & $0 / 6$ & $0 / 6$ & $0 / 6$ \\
\hline \multirow[t]{4}{*}{ Dullness } & $\mathrm{d} 0$ & $6 / 6$ & $6 / 6$ & $6 / 6$ \\
\hline & d 3 & $6 / 6$ & $4 / 6$ & $4 / 6$ \\
\hline & $\mathrm{d} 6$ & $4 / 6$ & $2 / 6$ & $2 / 6$ \\
\hline & $\mathrm{d} 9$ & $3 / 6$ & $1 / 6$ & $1 / 6$ \\
\hline
\end{tabular}


Table. 2 Changes in clinical vital parameters in cattle infected with T. annulata during 9 days of treatment period (Mean \pm S.E.)

\begin{tabular}{|c|c|c|c|c|}
\hline Parameter & Day post treatment & Group A $(n=6)$ & Group B $(n=6)$ & Group C $(n=6)$ \\
\hline \multirow{4}{*}{$\begin{array}{l}\text { Temperature } \\
\left({ }^{\circ} \mathrm{F}\right)\end{array}$} & $\mathrm{d} 0$ & $104.12 \pm 0.20^{\mathrm{ax}}$ & $104.57 \pm 0.16^{\mathrm{axy}}$ & $104.63 \pm 0.22^{\mathrm{ay}}$ \\
\hline & d 3 & $102.23 \pm 0.20^{\mathrm{b}}$ & $101.93 \pm 0.08^{b}$ & $102.27 \pm 0.12^{b}$ \\
\hline & d 6 & $102.00 \pm 0.10^{\mathrm{bx}}$ & $101.70 \pm 0.12^{\text {bxy }}$ & $101.43 \pm 0.12^{\text {cy }}$ \\
\hline & d 9 & $101.77 \pm 0.08^{b}$ & $101.67 \pm 0.20^{b}$ & $101.47 \pm 0.10^{\mathrm{c}}$ \\
\hline \multirow{4}{*}{$\begin{array}{l}\text { Pulse rate } \\
\text { (per min) }\end{array}$} & $\mathrm{d} 0$ & $68.00 \pm 2.59^{\mathrm{ax}}$ & $74.33 \pm 2.54^{\text {ay }}$ & $77.33 \pm 2.67^{\text {ay }}$ \\
\hline & d 3 & $74.67 \pm 1.96^{\mathrm{ax}}$ & $60.00 \pm 2.39^{\text {by }}$ & $63.50 \pm 2.74^{\text {by }}$ \\
\hline & $\mathrm{d} 6$ & $54.17 \pm 3.11^{\mathrm{b}}$ & $58.83 \pm 1.64^{\mathrm{b}}$ & $54.00 \pm 3.13^{\mathrm{c}}$ \\
\hline & d 9 & $48.00 \pm 1.37^{\mathrm{b}}$ & $45.00 \pm 2.38^{\mathrm{c}}$ & $42.67 \pm 2.47^{\mathrm{d}}$ \\
\hline \multirow{4}{*}{ Respiration rate (per min) } & $\mathrm{d} 0$ & $38.17 \pm 1.99^{\mathrm{ax}}$ & $47.33 \pm 1.45^{\text {ay }}$ & $47.67 \pm 2.70^{\text {ay }}$ \\
\hline & $\mathrm{d} 3$ & $45.33 \pm 1.52^{\mathrm{bx}}$ & $40.83 \pm 1.58^{\text {axy }}$ & $39.17 \pm 1.64^{\text {by }}$ \\
\hline & d 6 & $23.16 \pm 1.30^{\mathrm{c}}$ & $25.17 \pm 2.65^{b}$ & $20.33 \pm 1.23^{\mathrm{c}}$ \\
\hline & $\mathrm{d} 9$ & $19.67 \pm 1.28^{\mathrm{c}}$ & $20.33 \pm 30.50^{b}$ & $17.67 \pm 1.11^{\mathrm{c}}$ \\
\hline \multirow{4}{*}{$\begin{array}{l}\text { Lymph node size (scale } 1 \text { to } \\
\text { 4) }\end{array}$} & $\mathrm{d} 0$ & $2.50 \pm 0.22^{\mathrm{a}}$ & $2.83 \pm 0.31^{\mathrm{a}}$ & $3.17 \pm 0.31^{\mathrm{a}}$ \\
\hline & d 3 & $1.83 \pm 0.17^{\mathrm{b}}$ & $2.17 \pm 0.17^{\mathrm{b}}$ & $2.33 \pm 0.21^{\mathrm{b}}$ \\
\hline & d 6 & $1.83 \pm 0.17^{\mathrm{b}}$ & $1.83 \pm 0.17^{\mathrm{b}}$ & $1.83 \pm 0.17^{\mathrm{b}}$ \\
\hline & d 9 & $1.00 \pm 0.00^{\mathrm{c}}$ & $1.00 \pm 0.00^{\mathrm{c}}$ & $1.00 \pm 0.00^{\mathrm{c}}$ \\
\hline
\end{tabular}

Values with superscript $\mathrm{a}, \mathrm{b}, \mathrm{c}$ differ significantly $(\mathrm{p}<0.05)$ in a column and superscript $\mathrm{x}, \mathrm{y}$ differ significantly $(\mathrm{p}<0.05)$ in a row for a parameter

Table.3 Changes in haematological parameters in cattle infected with $T$. annulata during 9 days of treatment period (Mean \pm S.E.)

\begin{tabular}{|c|c|c|c|c|}
\hline Parameter & Day post treatment & Group A $(n=6)$ & Group B $(n=6)$ & Group C $(n=6)$ \\
\hline \multirow[t]{4}{*}{$\mathrm{Hb}(\mathrm{g} \%)$} & $\mathrm{d} 0$ & $8.63 \pm 0.57$ & $8.28 \pm 0.91$ & $7.48 \pm 0.20$ \\
\hline & d 3 & $8.38 \pm 0.53$ & $8.06 \pm 0.21$ & $7.18 \pm 0.33$ \\
\hline & $\mathrm{d} 6$ & $8.30 \pm 0.49$ & $7.72 \pm 0.27$ & $7.28 \pm 0.26$ \\
\hline & d 9 & $8.21 \pm 0.66$ & $8.00 \pm 0.35$ & $7.68 \pm 0.24$ \\
\hline \multirow[t]{4}{*}{ TEC $\left(\mathrm{M} / \mathrm{mm}^{3}\right)$} & $\mathrm{d} 0$ & $7.52 \pm 0.25$ & $6.58 \pm 0.31$ & $7.02 \pm 0.52$ \\
\hline & d 3 & $7.43 \pm 0.33$ & $6.48 \pm 0.25$ & $7.08 \pm 0.63$ \\
\hline & $\mathrm{d} 6$ & $7.37 \pm 0.36^{x y}$ & $6.39 \pm 0.22^{x}$ & $7.76 \pm 0.48^{y}$ \\
\hline & d 9 & $7.39 \pm 0.56$ & $6.62 \pm 0.27$ & $8.09 \pm 0.52$ \\
\hline \multirow[t]{4}{*}{ PCV (\%) } & $\mathrm{d} 0$ & $31.27 \pm 3.60$ & $32.57 \pm 2.52$ & $28.88 \pm 1.01$ \\
\hline & $\mathrm{d} 3$ & $33.35 \pm 1.87$ & $30.53 \pm 0.63$ & $28.93 \pm 0.75$ \\
\hline & $\mathrm{d} 6$ & $30.83 \pm 2.30$ & $29.97 \pm 0.64$ & $27.76 \pm 0.69$ \\
\hline & $\mathrm{d} 9$ & $32.78 \pm 2.81$ & $31.08 \pm 1.14$ & $30.05 \pm 0.66$ \\
\hline \multirow[t]{4}{*}{ TLC $\left(\mathrm{m} / \mathrm{mm}^{3}\right)$} & $\mathrm{d} 0$ & $6.76 \pm 0.96$ & $7.27 \pm 0.62$ & $7.14 \pm 1.08$ \\
\hline & d 3 & $6.86 \pm 0.93$ & $7.29 \pm 0.84$ & $7.33 \pm 1.21$ \\
\hline & $\mathrm{d} 6$ & $7.31 \pm 0.42$ & $7.67 \pm 0.87$ & $7.88 \pm 1.34$ \\
\hline & $\mathrm{d} 9$ & $7.45 \pm 0.85$ & $7.90 \pm 0.47$ & $7.95 \pm 1.35$ \\
\hline \multirow[t]{4}{*}{ Lymphocytes (\%) } & $\mathrm{d} 0$ & $81.95 \pm 8.78$ & $93.05 \pm 1.16^{\mathrm{a}}$ & $79.87 \pm 9.58$ \\
\hline & d 3 & $73.53 \pm 9.19$ & $88.47 \pm 1.30^{\mathrm{ab}}$ & $79.17 \pm 8.56$ \\
\hline & $\mathrm{d} 6$ & $82.20 \pm 7.51$ & $66.93 \pm 10.31^{\mathrm{bc}}$ & $70.35 \pm 10.63$ \\
\hline & $\mathrm{d} 9$ & $80.41 \pm 7.20$ & $59.40 \pm 10.85^{\mathrm{c}}$ & $69.36 \pm 10.03$ \\
\hline \multirow[t]{4}{*}{ Monocytes (\%) } & $\mathrm{d} 0$ & $5.98 \pm 2.99$ & $1.60 \pm 0.21^{\mathrm{a}}$ & $7.05 \pm 3.65$ \\
\hline & $\mathrm{d} 3$ & $8.98 \pm 3.43$ & $2.58 \pm 0.29^{\mathrm{a}}$ & $7.30 \pm 3.57$ \\
\hline & $\mathrm{d} 6$ & $6.95 \pm 3.40$ & $6.07 \pm 1.89^{\mathrm{ab}}$ & $7.13 \pm 3.30$ \\
\hline & d 9 & $8.67 \pm 2.99$ & $10.87 \pm 2.7^{b}$ & $7.48 \pm 3.37$ \\
\hline \multirow[t]{4}{*}{ Neutrophils (\%) } & $\mathrm{d} 0$ & $10.42 \pm 5.40$ & $2.68 \pm 0.35^{\mathrm{a}}$ & $11.92 \pm 5.82$ \\
\hline & d 3 & $15.03 \pm 5.77$ & $7.15 \pm 1.64^{\mathrm{ab}}$ & $12.85 \pm 5.62$ \\
\hline & d 6 & $9.20 \pm 3.64$ & $18.93 \pm 7.25^{b}$ & $6.60 \pm 3.15$ \\
\hline & d 9 & $11.82 \pm 3.87$ & $20.37 \pm 6.49^{b}$ & $11.10 \pm 3.83$ \\
\hline \multirow[t]{4}{*}{ Eosinophils (\%) } & $\mathrm{d} 0$ & $1.45 \pm 0.59$ & $2.52 \pm 1.16$ & $1.07 \pm 0.62^{\mathrm{a}}$ \\
\hline & d 3 & $2.27 \pm 1.02$ & $1.68 \pm 1.06$ & $0.67 \pm 0.67^{\mathrm{a}}$ \\
\hline & $\mathrm{d} 6$ & $1.55 \pm 0.79$ & $7.58 \pm 3.66$ & $5.62 \pm 2.27^{b}$ \\
\hline & d 9 & $5.55 \pm 2.28^{x y}$ & $8.92 \pm 2.24^{x}$ & $1.90 \pm 1.05^{\text {aby }}$ \\
\hline \multirow[t]{4}{*}{ Basophils (\%) } & $\mathrm{d} 0$ & $0.17 \pm 0.08$ & $0.15 \pm 0.08$ & $0.10 \pm 0.05^{\mathrm{ab}}$ \\
\hline & d 3 & $0.18 \pm 0.07$ & $0.72 \pm 0.66$ & $0.02 \pm 0.02^{\mathrm{a}}$ \\
\hline & d 6 & $0.10 \pm 0.04$ & $0.38 \pm 0.11$ & $0.28 \pm 0.19^{\mathrm{b}}$ \\
\hline & d 9 & $0.25 \pm 0.09$ & $0.35 \pm 0.14$ & $0.15 \pm 0.07^{\mathrm{ab}}$ \\
\hline
\end{tabular}

Values with superscript $\mathrm{a}, \mathrm{b}$, c differ significantly $(\mathrm{p}<0.05)$ in a column and superscript $\mathrm{x}$, y differ significantly $(\mathrm{p}<0.05)$ in a row for a parameter

$\mathrm{Hb}=$ Haemoglobin content; TEC=Total erythrocyte count; PCV=Packed cell volume; TLC=Total leucocyte count. 
Table.4 Changes in liver function tests in cattle infected with $T$. annulata during 9 days of treatment period (Mean \pm S.E.)

\begin{tabular}{|c|c|c|c|c|}
\hline Serum biochemical parameter & $\begin{array}{l}\text { Day post } \\
\text { treatment }\end{array}$ & $\begin{array}{l}\text { Group A } \\
(n=6)\end{array}$ & $\begin{array}{l}\text { Group B } \\
(\mathrm{n}=6)\end{array}$ & $\begin{array}{l}\text { Group C } \\
(\mathrm{n}=6)\end{array}$ \\
\hline \multirow{4}{*}{$\overline{\mathrm{ALT}}(\mathrm{U} / \mathrm{L})$} & $\mathrm{d} 0$ & $35.90 \pm 2.16^{\mathrm{a}}$ & $38.05 \pm 3.32^{\mathrm{a}}$ & $36.20 \pm 3.12^{\mathrm{a}}$ \\
\hline & d 3 & $30.30 \pm 2.51^{\mathrm{abx}}$ & $30.13 \pm 1.69^{b x}$ & $19.72 \pm 3.21^{\text {by }}$ \\
\hline & $\mathrm{d} 6$ & $29.45 \pm 2.11^{\mathrm{abx}}$ & $28.33 \pm 1.29^{\text {bx }}$ & $13.58 \pm 3.95^{\text {by }}$ \\
\hline & d 9 & $28.48 \pm 2.41^{\mathrm{bx}}$ & $27.83 \pm 0.94^{\mathrm{bx}}$ & $12.23 \pm 0.80^{\text {by }}$ \\
\hline \multirow[t]{4}{*}{ AST (U/L) } & $\mathrm{d} 0$ & $65.63 \pm 0.70$ & $80.35 \pm 17.48$ & $70.15 \pm 5.15$ \\
\hline & d 3 & $51.67 \pm 7.66$ & $73.54 \pm 14.62$ & $65.58 \pm 5.01$ \\
\hline & $\mathrm{d} 6$ & $50.49 \pm 6.13$ & $66.82 \pm 9.19$ & $57.58 \pm 11.30$ \\
\hline & d 9 & $48.17 \pm 5.91$ & $59.80 \pm 6.98$ & $48.52 \pm 7.08$ \\
\hline \multirow[t]{4}{*}{ GGT (U/L) } & $\mathrm{d} 0$ & $18.32 \pm 0.80^{x}$ & $24.65 \pm 2.61^{x}$ & $33.38 \pm 3.14^{y}$ \\
\hline & d 3 & $18.46 \pm 3.29$ & $20.06 \pm 2.61$ & $30.87 \pm 5.76$ \\
\hline & $\mathrm{d} 6$ & $17.70 \pm 3.31$ & $17.28 \pm 3.21$ & $29.82 \pm 5.98$ \\
\hline & d 9 & $17.60 \pm 2.12^{\mathrm{xy}}$ & $16.46 \pm 1.64^{x}$ & $28.43 \pm 5.62^{y}$ \\
\hline \multirow{4}{*}{ Bilirubin total $(\mathrm{mg} / \mathrm{dl})$} & $\mathrm{d} 0$ & $0.15 \pm 0.02$ & $0.13 \pm 0.02$ & $0.21 \pm 0.04^{\mathrm{a}}$ \\
\hline & d 3 & $0.17 \pm 0.03^{\mathrm{x}}$ & $0.09 \pm 0.01^{\mathrm{y}}$ & $0.12 \pm 0.02^{\mathrm{bxy}}$ \\
\hline & $\mathrm{d} 6$ & $0.13 \pm 0.01$ & $0.13 \pm 0.01$ & $0.12 \pm 0.01^{b}$ \\
\hline & d 9 & $0.12 \pm 0.01$ & $0.10 \pm 0.01$ & $0.09 \pm 0.01^{b}$ \\
\hline \multirow{4}{*}{ Bilirubin direct $(\mathrm{mg} / \mathrm{dl})$} & $\mathrm{d} 0$ & $0.12 \pm 0.02$ & $0.09 \pm 0.01^{\mathrm{a}}$ & $0.11 \pm 0.02$ \\
\hline & d 3 & $0.11 \pm 0.02^{\mathrm{x}}$ & $0.05 \pm 0.01^{\text {by }}$ & $0.08 \pm 0.01^{\mathrm{xy}}$ \\
\hline & $\mathrm{d} 6$ & $0.09 \pm 0.01$ & $0.08 \pm 0.01^{\mathrm{a}}$ & $0.07 \pm 0.02$ \\
\hline & d 9 & $0.09 \pm 0.01$ & $0.07 \pm 0.01^{\mathrm{ab}}$ & $0.06 \pm 0.01$ \\
\hline \multirow[t]{4}{*}{ Bilirubin indirect $(\mathrm{mg} / \mathrm{dl})$} & $\mathrm{d} 0$ & $0.03 \pm 0.00^{\mathrm{abx}}$ & $0.03 \pm 0.01^{\mathrm{x}}$ & $0.10 \pm 0.03^{\text {ay }}$ \\
\hline & d 3 & $0.06 \pm 0.02^{\mathrm{b}}$ & $0.04 \pm 0.01$ & $0.04 \pm 0.01^{b}$ \\
\hline & $\mathrm{d} 6$ & $0.03 \pm 0.01^{\mathrm{ab}}$ & $0.05 \pm 0.01$ & $0.05 \pm 0.01^{b}$ \\
\hline & d 9 & $0.03 \pm 0.01^{\mathrm{a}}$ & $0.05 \pm 0.01$ & $0.03 \pm 0.01^{b}$ \\
\hline
\end{tabular}

Values with superscript $\mathrm{a}, \mathrm{b}$ differ significantly $(\mathrm{p}<0.05)$ in a column and superscript $\mathrm{x}$, y differ significantly $(\mathrm{p}<0.05)$ in a row for a parameter

ALT=Alanine aminotransaminase; AST=aspartate aminotransaminase; GGT=Gamma glutamyl transferase.

Table.5 Changes in protein profile in cattle infected with $T$. annulata during 9 days of treatment period (Mean \pm S.E.)

\begin{tabular}{|l|l|l|l|l|}
\hline Serum biochemical parameter & $\begin{array}{l}\text { Day post } \\
\text { treatment }\end{array}$ & $\begin{array}{l}\text { Group A } \\
(\mathrm{n}=6)\end{array}$ & $\begin{array}{l}\text { Group B } \\
(\mathrm{n}=6)\end{array}$ & $\begin{array}{l}\text { Group C } \\
(\mathrm{n}=6)\end{array}$ \\
\hline \multirow{5}{*}{ Total protein $(\mathrm{g} / \mathrm{dl})$} & $\mathrm{d} 0$ & $6.02 \pm 0.94^{\mathrm{a}}$ & $7.40 \pm 0.33^{\mathrm{a}}$ & $5.83 \pm 0.56^{\mathrm{a}}$ \\
\cline { 2 - 5 } & $\mathrm{d} 3$ & $8.00 \pm 0.38^{\mathrm{b}}$ & $8.05 \pm 0.35^{\mathrm{ab}}$ & $8.10 \pm 0.49^{\mathrm{b}}$ \\
\hline & $\mathrm{d} 6$ & $8.15 \pm 0.35^{\mathrm{b}}$ & $8.34 \pm 0.36^{\mathrm{ab}}$ & $8.35 \pm 0.51^{\mathrm{b}}$ \\
\hline & $\mathrm{d} 9$ & $8.35 \pm 0.28^{\mathrm{b}}$ & $8.58 \pm 0.31^{\mathrm{b}}$ & $8.55 \pm 0.39^{\mathrm{b}}$ \\
\hline \multirow{5}{*}{ Albumin $(\mathrm{g} / \mathrm{dl})$} & $\mathrm{d} 0$ & $3.13 \pm 0.12$ & $2.82 \pm 0.20$ & $2.90 \pm 0.19$ \\
\cline { 2 - 5 } & $\mathrm{d} 3$ & $3.20 \pm 0.08$ & $3.19 \pm 0.18$ & $2.92 \pm 0.18$ \\
\hline & $\mathrm{d} 6$ & $3.31 \pm 0.12$ & $3.27 \pm 0.18$ & $2.98 \pm 0.13$ \\
\cline { 2 - 5 } & $\mathrm{d} 9$ & $3.33 \pm 0.12$ & $3.35 \pm 0.14$ & $3.00 \pm 0.15$ \\
\hline \multirow{5}{*}{ Globulin $(\mathrm{g} / \mathrm{dl})$} & $\mathrm{d} 0$ & $2.88 \pm 0.89^{\mathrm{a}}$ & $4.58 \pm 0.41$ & $2.93 \pm 0.66^{\mathrm{a}}$ \\
\cline { 2 - 5 } & $\mathrm{d} 3$ & $4.80 \pm 0.37^{\mathrm{b}}$ & $4.86 \pm 0.40$ & $5.18 \pm 0.63^{\mathrm{b}}$ \\
\hline & $\mathrm{d} 6$ & $4.84 \pm 0.35^{\mathrm{b}}$ & $5.07 \pm 0.40$ & $5.37 \pm 0.60^{\mathrm{b}}$ \\
\hline & $\mathrm{d} 9$ & $5.02 \pm 0.34^{\mathrm{b}}$ & $5.23 \pm 0.39$ & $5.55 \pm 0.41^{\mathrm{b}}$ \\
\hline
\end{tabular}

Values with superscript $a, b$ differ significantly $(\mathrm{p}<0.05)$ in a column 
Table.6 Changes in oxidative stress indices (in plasma and hemolysate) in cattle infected with $T$. annulata during 9 days of treatment period (Mean \pm S.E.)

\begin{tabular}{|c|c|c|c|c|}
\hline Parameter & $\begin{array}{l}\text { Day post } \\
\text { treatment }\end{array}$ & $\begin{array}{l}\text { Group A } \\
(\mathrm{n}=6)\end{array}$ & $\begin{array}{l}\text { Group B } \\
(\mathrm{n}=6)\end{array}$ & $\begin{array}{l}\text { Group C } \\
(\mathrm{n}=6)\end{array}$ \\
\hline \multirow[t]{4}{*}{ MDA plasma $(\mathrm{nmol} / \mathrm{ml})$} & $\mathrm{d} 0$ & $17.06 \pm 3.01$ & $13.87 \pm 1.30^{\mathrm{a}}$ & $19.13 \pm 5.17$ \\
\hline & d 3 & $20.48 \pm 4.79$ & $9.79 \pm 1.34^{b}$ & $15.00 \pm 5.29$ \\
\hline & d 6 & $23.88 \pm 5.40^{x}$ & $6.84 \pm 1.40^{\text {bcy }}$ & $9.74 \pm 4.48^{y}$ \\
\hline & d 9 & $26.42 \pm 5.06^{\mathrm{x}}$ & $5.46 \pm 0.61^{\mathrm{cy}}$ & $5.45 \pm 1.06^{\mathrm{y}}$ \\
\hline \multirow{4}{*}{$\begin{array}{l}\text { MDA haemolysate } \\
(\mathrm{nmol} / \mathrm{ml})\end{array}$} & $\mathrm{d} 0$ & $38.16 \pm 7.76$ & $40.35 \pm 1.63^{\mathrm{a}}$ & $42.25 \pm 4.04^{\mathrm{a}}$ \\
\hline & d 3 & $48.24 \pm 8.73$ & $36.63 \pm 2.04^{\mathrm{a}}$ & $36.13 \pm 3.11^{\mathrm{ab}}$ \\
\hline & $\mathrm{d} 6$ & $56.16 \pm 10.08^{x}$ & $32.27 \pm 4.89^{\text {aby }}$ & $24.64 \pm 4.97^{\text {bcy }}$ \\
\hline & d 9 & $63.29 \pm 7.14^{x}$ & $24.61 \pm 4.05^{\text {by }}$ & $17.13 \pm 4.45^{\mathrm{cy}}$ \\
\hline \multirow{4}{*}{$\begin{array}{l}\text { GPx plasma }(\mathrm{U} / \mathrm{mg} \\
\text { protein) }\end{array}$} & $\mathrm{d} 0$ & $3.18 \pm 0.53^{\mathrm{a}}$ & $3.45 \pm 0.20^{\mathrm{a}}$ & $3.79 \pm 0.21^{\mathrm{a}}$ \\
\hline & d 3 & $3.87 \pm 0.29^{\mathrm{abx}}$ & $2.44 \pm 0.46^{\text {by }}$ & $1.90 \pm 0.29^{\mathrm{by}}$ \\
\hline & d 6 & $4.03 \pm 0.56^{\mathrm{abx}}$ & $2.29 \pm 0.27^{\text {by }}$ & $1.85 \pm 0.35^{\text {by }}$ \\
\hline & d 9 & $4.79 \pm 0.51^{\mathrm{bx}}$ & $2.15 \pm 0.12^{\text {by }}$ & $0.94 \pm 0.11^{\mathrm{cz}}$ \\
\hline \multirow{4}{*}{$\begin{array}{l}\text { GPx haemolysate }(\mathrm{U} / \mathrm{mg} \\
\mathrm{Hb})\end{array}$} & $\mathrm{d} 0$ & $6.06 \pm 1.20^{\mathrm{a}}$ & $6.81 \pm 1.47$ & $7.68 \pm 1.03^{\mathrm{a}}$ \\
\hline & d 3 & $7.92 \pm 0.57^{\mathrm{abx}}$ & $6.47 \pm 1.29^{\mathrm{xy}}$ & $4.62 \pm 0.93^{\text {by }}$ \\
\hline & $\mathrm{d} 6$ & $9.48 \pm 1.33^{\mathrm{bx}}$ & $5.16 \pm 0.62^{y}$ & $3.56 \pm 1.24^{\text {by }}$ \\
\hline & d 9 & $10.20 \pm 1.12^{\mathrm{bx}}$ & $3.85 \pm 0.30^{y}$ & $1.92 \pm 0.83^{\text {by }}$ \\
\hline \multirow{4}{*}{$\begin{array}{l}\text { SOD plasma }(\mathrm{U} / \mathrm{mg} \\
\text { protein) }\end{array}$} & $\mathrm{d} 0$ & $0.38 \pm 0.01$ & $0.44 \pm 0.02^{\mathrm{a}}$ & $0.50 \pm 0.08^{\mathrm{a}}$ \\
\hline & $\mathrm{d} 3$ & $0.38 \pm 0.02$ & $0.32 \pm 0.04^{\mathrm{ab}}$ & $0.36 \pm 0.02^{b}$ \\
\hline & $\mathrm{d} 6$ & $0.40 \pm 0.02$ & $0.28 \pm 0.06^{\mathrm{b}}$ & $0.34 \pm 0.02^{b}$ \\
\hline & d 9 & $0.41 \pm 0.02^{\mathrm{x}}$ & $0.23 \pm 0.05^{\text {by }}$ & $0.30 \pm 0.02^{\text {by }}$ \\
\hline \multirow{4}{*}{$\begin{array}{l}\text { SOD haemolysate (U/mg } \\
\mathrm{Hb})\end{array}$} & $\mathrm{d} 0$ & $0.12 \pm 0.01^{\mathrm{x}}$ & $0.26 \pm 0.05^{\mathrm{ay}}$ & $0.16 \pm 0.02^{\mathrm{axy}}$ \\
\hline & d 3 & $0.12 \pm 0.02$ & $0.16 \pm 0.04^{\mathrm{ab}}$ & $0.11 \pm 0.0^{b}$ \\
\hline & $\mathrm{d} 6$ & $0.13 \pm 0.01$ & $0.14 \pm 0.02^{\mathrm{b}}$ & $0.09 \pm 0.02^{\mathrm{b}}$ \\
\hline & d 9 & $0.15 \pm 0.02^{\mathrm{x}}$ & $0.12 \pm 0.02^{\mathrm{bx}}$ & $0.07 \pm 0.01^{\text {by }}$ \\
\hline
\end{tabular}

Values with superscript a, b, c differ significantly $(\mathrm{p}<0.05)$ in a column and superscript $\mathrm{x}, \mathrm{y}, \mathrm{z}$ differ significantly $(\mathrm{p}<0.05)$ in a row for a parameter

MDA=Malondialdehyde level; GPx=Glutathione peroxidase activity; SOD=Superoxide dismutase activity.

\section{Serological examinations}

Changes in liver function tests are depicted in table 4. Significant $(\mathrm{p} \leq 0.05)$ decrease in levels of ALT in all groups was observed and among groups maximum decrease in animals of group $\mathrm{C}$ was found. Along with it, total bilirubin and indirect bilirubin decreased significantly in group C. Rest of the parameters altered non-significantly. However slight decrease in levels of other hepatic enzymes was observed in all groups with maximum decrease in group C. In group A, it may be due to the removal of parasitic burden on hepatocytes, which were deteriorating the functions of hepatocytes. In group $\mathrm{B}$ and $\mathrm{C}$ improved functions of liver may be due to ability of vitamin $\mathrm{C}$ and vitamin E-selenium to protect the hepatic cells from oxidative damage and lipid peroxidation, which is mediated by oxygenfree radicals. The protective effect may be due to stabilization of plasma membrane thereby preserving the structural integrity of hepatocytes.

\section{Protein profile}

Changes in protein profile is depicted in table 5 . Total protein increased significantly $(p \leq$ 0.05 ) in animals of all groups. Globulin increased significantly in group A and group C. Albumin level increased non-significantly in all groups. These findings might be due to 
the improvement in hepatic function which leads to improvement in biosynthesis of proteins.

Evaluation of oxidative stress and antioxidants in response to therapy

Oxidative stress indices in terms of MDA, GPx and SOD measured in plasma and haemolysate, are depicted in table 6 .

\section{MDA levels}

MDA was found to be gradually increased, both in plasma and haemolysate from day 0 to day 9 in group A. This finding is in agreement with Naziroglu et al., (1999) and Kumar et al., (2016). This finding might be due to buparvaquone administration which might function by forming free radicals to kill $T$. annulata infection. McHardy (1989) also reported that buparvaquone persists for a long time in plasma (half-life 7 days). Hence, elevated level of MDA after treatment with buparvaquone in the present study might be due to residual effect of buparvaquone in body of animals. Vitamin $\mathrm{C}$ and vitamin $\mathrm{E}$ selenium supplementation caused significant decrease in level of MDA (lipid peroxidation) both, in plasma and haemolysate. Vitamin C acts as an inhibitor/chain blocker of lipid peroxidation (Tanaka et al., 2007). Vitamin E-selenium provides protection against damaging effect of free radicals on cellular components (Papas, 1999). Therefore, the decrease in the level of MDA might be due to the reduction in cellular damage caused by free radicals due to supplementation with antioxidants.

\section{GPx levels}

In group A, level of GPx increased significantly both in plasma and haemolysate from day 0 to day 9 . It might be due to the fact that, animal body was still under oxidative stress and levels of GPx increased to combat the effects of free radicals. While antioxidant therapy significantly decreased the level of GPx in group B and group C (both, in plasma and haemolysate) indicating that the oxidative stress was reduced in both groups. As compared to group B significant decrease in GPx levels in plasma was found in animals of group C. As Vitamin Eselenium is the important part of antioxidant defense system of living tissues (Gerloff, 1992). Vitamin $E$ has the capacity to quench free radicals which causes the initiation and propagation of lipid oxidation, and the Secontaining antioxidant enzyme (GPx) catalyses the decomposition of lipid hydroperoxides into less-reactive products (DeVore et al., 1983, Faustman et al., 1989). Glutathione peroxidase a selenoenzymes, and the Se status of the body influences its activity (Brown and Arthur, 2001).

\section{SOD levels}

SOD level non-significantly increased from day 0 to day 9 in group A. In animals of group $B$ and group $C$ which were administered antioxidant therapy, level of SOD were significantly lower after treatment in both plasma and haemolysate. SOD level in haemolysate was significantly lower in group $\mathrm{C}$ as compared to group $\mathrm{B}$. The activity of enzyme SOD could also be assigned as a major factor in preventing the $\mathrm{RBC}$ membrane from peroxide damage, induced by lipid peroxidation.

Antioxidants (vitamin C and vitamin Eselenium) greatly help in recovery of infected animals from theileriosis and leads to fast clinical recovery when supplemented with specific drug therapy. Antioxidants greatly help in reduction of damage caused by free radicals and plays important role in improvement in liver functions. Vitamin Eselenium given 3 times at 3 days interval 
proved to be better antioxidant therapy as compared to vitamin $\mathrm{C}$ given every day for 9 days. So antioxidants should be given as adjunct therapy for treatment of bovine tropical theileriosis for fast clinical recovery of animals.

\section{References}

Al-Robayi, H.M. 1999. Epidemiology of Theileria annulata infection in with $\mathrm{Al}$ Ashaiki farm Doctoral dissertation, $\mathrm{Ph}$. D. Thesis., College of Veterinary Medicine, University of Baghdad. (In Arabic). pp: 29-74.

Al-Temeimy, D.A.H.J. 1982. Some studies on calves experimentally infected with Theileria annulata. M. Sc. Thesis., College of Veterinary Medicine, University of Baghdad. (In Arabic). pp:20-87.

Bast, A., Haenen G.R. and Doelman, C.J. 1991. Oxidants and antioxidants: state of the art. The American Journal of Medicine. 91(3): S2-13.

Brown, K.M. and Arthur, J.R. 2001. Selenium, selenoproteins and human health: a review. Public Health Nutrition. 4: 593-599.

DeVore, V.R., Colnago, G.L., Jensen, L.S. and Greene, B.E. 1983. Thiobarbituric Acid Values and Glutathione Perioxidase Activity in Meat from Chickens Fed a Selenium- Supplemented Diet. Journal of Food Science. 48(1): 300-306.

Dhar, S., Malhotra, D.V., Bhushan, C. and Gautam, O.P. 1988. Treatment of experimentally induced Theileria annulata infection in cross-bred calves with buparvaquone. Veterinary Parasitology. 27(3): 267-275.

El-Deeb, W.M. and Younis, E.E. 2009. Clinical and biochemical studies on Theileria annulata in Egyptian buffaloes (Bubalus bubalis) with particular orientation to oxidative stress and ketosis relationship. Veterinary Parasitology. 164(2): 301-305.

Faustman, C., Cassens, R.G., Schaefer, D.M., Buege, D.R., Williams, S.N. and Scheller, K.K. 1989. Improvement of pigment and lipid stability in Holstein steer beef by dietary supplementation with vitamin E. Journal of Food Science. 54(4): 858-862.

Gerloff, B.J. 1992. Effect of selenium supplementation on dairy cattle. Journal of Animal Science. 70(12): 3934-3940.

Grewal, A., Ahuja, C.S., Singha, S.P. and Chaudhary, K.C. 2005. Status of lipid peroxidation, some antioxidant enzymes and erythrocytic fragility of crossbred cattle naturally infected with Theileria annulata. Veterinary Research Communications. 29(5):387-94.

Hafeman, D.G., Sunde, R.A. and Hoekstra, W.G. 1974. Effect of dietary selenium on erythrocyte and liver glutathione peroxidase in the rat. The Journal of Nutrition. 104(5):580-587.

Issi, M. and Gul, Y. 2001. Cited in Altug, N., Yuksek, N., Keles, I., Ozkan, C., Yoruk, I.H. and Arslan, S. 2014. Efficiency of various supportive treatments as a cure for anaemia in cattle with theileriosis. Thailand Journal of Veterinary Medicine. 44(3): 287-296.

Keleş. İ., Değer, S., Altuğ, N., Karaca, M. and Akdemir, C. 2001. Tick-borne diseases in cattle: Clinical and haematological findings, diagnosis, treatment, seasonal distribution, breed, sex and age factors and the transmitters of the diseases. YYU Vet Fak Derg. 12: 26-32.

Kumar, A., Rakha, N.K. and Virmani, M. 2016. Enhancing clinical recovery of bovine tropical theileriosis by use of antioxidant supportive therapy. The Indian Journal of Animal Sciences. 86(5): 518-524.

Kumar, R. and Malik, J.K. 1999. Influence of 
experimentally induced theileriosis (Theileria annulata) on the pharmacokinetics of a long-acting formulation of oxytetracycline (OTCLA) in calves. Journal of Veterinary Pharmacology and Therapeutics. 22: 320-326.

Madesh, M. and Balasubramanian, K.A. 1998. Microtiter plate assay for superoxide dismutase using MTT reduction by superoxide. Indian Journal of Biochemistry and Biophysics. 35(3):184-188.

Mbassa, G.K., Balemba, O., Maselle, R.M. and Mwaga, N.V. 1994. Severe anaemia due to haematopoietic precursor cell destruction in field cases of East Coast Fever in Tanzania. Veterinary Parasitology. 52: 243-256.

McHardy, N. 1989. Buparvaquone, the new antitheilerial: a review of its efficacy and safety. In: Dolan, T.T. (Ed.), Theileriosis in Eastern, Central and Southern Africa. Proc. Workshop on East Coast Fever Immunisation held in Lilongwe, Malawi, 20- 22 September 1988. ILRAD, Nairobi, pp. 158-165.

McHardy, N. 1990. Butalex (buparvaquone): A new therapeutic for theileriosis. Proceedings of a Workshop Held at Ilrad Nairobi (Kenya). 59-66.

Nazifi, S., Razavi, S.M., Hasanshahi, F. and Esmailnezhad, Z. 2009. Effect of the severity of Theileria annulata infection on some haematological parameters and antioxidant enzymes in naturally infected cattle. Bulgarian Journal of Veterinary Medicine. 12(1):63-71.

Nazifi, S., Razavi, S.M., Mansourian, M., Nikahval, B. and Moghaddam, M. 2008. Studies on correlations among parasitaemia and some hemolytic indices in two tropical diseases (theileriosis and anaplasmosis) in Fars province of Iran. Tropical Animal Health and Production. 40(1): 47-53.
Naziroğlu, M., Saki, C.E. and Sevgili, M. 1999. The effect of buparvaquone treatment on the levels of some antioxidant vitamins, lipid peroxidation and glutathione peroxidase in cattle with theileriosis. Journal of Veterinary Medicine. 46(4): 233-239.

Ogutcu, A., Uzunhisarcikli, M., Kalender, S., Durak, D., Bayrakdar, F. and Kalender, Y. 2006. The effects of organophosphate insecticide diazinon on malondialdehyde levels and myocardial cells in rat heart tissue and protective role of vitamin E. Pesticide Biochemistry and Physiology. 86(2): 93-98.

Ohkawa, H., Ohishi, W. and Yagi, K. 1979. Lipid peroxidation (malondialdehyde). Colorimetric method. Analytical Biochemistry. 95:351.

Omer, O.H., El-Malik, K.H., Mahmoud, O.M., Haroun, E.M., Hawas, A., Sweeney, D. and Magzoub, M. 2002. Haematological profiles in pure bred cattle naturally infected with Theileria annulata in Saudi Arabia. Veterinary Parasitology. 107(1):161-168.

Papas, A.M. 1999. Diet and antioxidant status. Food and Chemical Toxicology. 37(9): 999-1007.

Preston, P.M., Jackson, L.A., Sutherland, I.A., Brown, D.J., Schofield, J., Bird, T., Sanderson, A. and Brown, C.G. 2001. Theileria annulata: Attenuation of a schizont-infected cell line by prolonged in vitro culture is not caused by the preferential growth of particular host cell types. Experimental Parasitology. 98(4):188-205.

Rakha, N.K. and Sharma, R.D. 2003. Current epidemiological status of bovine tropical theileriosis in Haryana (India). Intas Polivet. 4(1): 15-22.

Saleh, M.A., Mahran, O.M. and Al-Salahy, M.B. 2011. Corpuscular oxidation in newborn crossbred calves naturally 
infected with Theileria annulata. Veterinary Parasitology. 182(2):193200.

Sharma, L.D., Sharma, N.N., Sabir, M., Bhattacharyya, N.K. 1987. Clinicohaematological and biochemical changes in experimental Theileria infection in crossbred calves. Indian Journal of Veterinary Medicine. 7:154156.

Shiono, H., Yagi, Y., Kumar, A., Yamanaka, M. and Chikayama, Y. 2004. Accelerated binding of autoantibody to red blood cells with increasing anaemia in cattle experimentally infected with Theileria sergenti. Journal of Veterinary Medicine. 51(1):39-42.

Shiono, H., Yagi. Y., Thongnoon, P., Kurabayashi, N., Chikayama, Y., Miyazaki, S. and Nakamura, I. 2001. Acquired methemoglobinemia in anemic cattle infected with Theileria sergenti. Veterinary Parasitology. 102(1):45-51.

Singh, D.K. 1990. Methods currently used for the control of Theileria annulata: their validity and proposals for future control strategies. Parassitologia. 32(1): 33.

Smith, K.L., Hogan, J.S. and Weiss, W.P. 1997. Dietary vitamin E and selenium affect mastitis and milk quality. Journal of Animal Sciences. 75: 1659-1665.

Stockham, S.L., Kjemtrup, A.M., Conrad, P.A., Schmidt, D.A., Scott, M.A., Robinson, T.W., Tyler, J.W., Johnson, G.C., Carson, C.A. and Cuddihee, P. 2000. Theileriosis in a Missouri beef herd caused by Theileria buffeli: case report, herd investigation, ultrastructure, phylogenetic analysis, and experimental transmission. Veterinary Pathology. 37(1):11-21.

Tanaka, M., Kamiya, Y., Kamiya, M. and Nakai, Y. 2007. Effect of high environmental temperatures on ascorbic acid, sulfydryl residues and oxidized lipid concentrations in plasma of dairy cows. Journal of Animal Science. 78: 301-306.

Valko, M., Rhodes, C.J., Moncol, J., Izakovic, M.M. and Mazur, M. 2006. Free radicals, metals and antioxidants in oxidative stress-induced cancer. Chemico-biological Interactions. 160(1):1-40.

Vankampen, E.J., and Ziglstra, W.G. 1961. Colorimetric determination of haemoglobin Clinica Chemica Acta 6 p. 5388.

\section{How to cite this article:}

Neelam, Naresh Kumar Rakha, Ricky Jhambh, Meenakshi Virmani, Parveen Goel and Ramkaran. 2017. Evaluation of the Antioxidants as Adjunct Therapy in Cattle Naturally Infected with Bovine Tropical Theileriosis. Int.J.Curr.Microbiol.App.Sci. 6(11): 5373-5384. doi: https://doi.org/10.20546/ijcmas.2017.611.514 